\title{
Treatment of Primary Insomnia
}

\author{
Erika N. Ringdabl, MD, Susan L. Pereira, MD, and John E. Delzell, Jr, MD, MSPH
}

Ten percent to $40 \%$ of adults have intermittent insomnia, and $15 \%$ have long-term sleep difficulties. This article provides a review of the classification, differential diagnosis, and treatment options available for insomnia. We performed a MEDLINE search using OVID and the key words "insomnia," "sleeplessness," "behavior modification," "herbs," "medicinal," and "pharmacologic therapy." Articles were selected based on their relevance to the topic. Evaluation of insomnia includes a careful sleep history, review of medical history, review of medication use (including over-the-counter and herbal medications), family history, and screening for depression, anxiety, and substance abuse. Treatment should be individualized based on the nature and severity of symptoms. Nonpharmacologic treatments are effective and have minimal side effects compared with drug therapies. Medications such as diphenhydramine, doxylamine, and trazodone can be used initially, but patients may not tolerate their side effects. Newer medications such as zolpidem and zaleplon have short half-lives and minimal side effects. Both are approved for short-term use in the insomniac. (J Am Board Fam Pract 2004;17:212-9.)

Many people have difficulty sleeping. A 1995 Gallup survey found that $49 \%$ of adults were dissatisfied with their sleep at least 5 nights per month. ${ }^{1}$ Population-based studies estimate that $10 \%$ to $40 \%$ of American adults have intermittent insomnia; $10 \%$ to $15 \%$ have long-term sleep difficulties. ${ }^{2}$ Insomnia has been associated with decreases in work performance and increases in motor vehicle accidents and hospitalization rates. ${ }^{3}$ Cost estimates for lost productivity and insomnia-related accidents exceed $\$ 100$ billion per year. ${ }^{4}$ The purpose of this review is to provide a current review of the classification, the differential diagnosis, and treatment options for insomnia. We performed a MEDLINE search using OVID and the key words "insomnia," "sleeplessness," "behavior modification," "herbs," "medicinal," and "pharmacologic therapy." Abstracts were reviewed by 2 of the authors (ENR, SLP). Articles were then selected based on relevance to the topical review.

Submitted, revised, 24 December 2003.

From the Departments of Family and Community Medicine (ENR) and Urgent Care (SLP) University of MissouriColumbia School of Medicine, Columbia, and the Department of Family Medicine, University of Tennessee College of Medicine, Memphis. Address correspondence to Erika Ringdahl, MD, Family Practice Residency Program, Department of Family and Community Medicine, MA303 Medical Sciences Building, Columbia, MO 65212 (e-mail: ringdahle@health.missouri.edu).

\section{Classification}

The American Psychiatric Association's Diagnostic and Statistical Manual of Mental Disorders (DSM-IV) ${ }^{5}$ defines insomnia as a complaint regarding the quantity, quality, or sleep timing at least 3 times a week for at least 1 month. Sleep is divided into rapid eye movement (REM) sleep and non-REM sleep. Non-REM sleep has 4 stages, each progressively deeper. Stages 3 and 4, deep restorative sleep, are also called slow wave or $\delta$ sleep. Decreased time spent in stage 3 and 4 decreases sleep quality. Stage 5 sleep is REM sleep. ${ }^{6}$ Research studies define insomnia as a sleep latency (time taken to fall asleep) that is greater than 30 minutes, sleep efficiency (time asleep/time in bed) less than $85 \%$, or sleep disturbance more than 3 times a week. ${ }^{7}$

The International Classification of Sleep Disorders classifies insomnia as a dyssomnia. ${ }^{8}$ Dyssomnias include difficulties initiating or maintaining sleep (insomnia) and excessive sleep (hypersomnia). Several insomnia-related complaints defy categorization. $\mathrm{Pa}-$ tients who have sleep state misperception insomnia complain of not having slept for a long time without objective evidence of difficulty sleeping. Some patients sleep less because of work or social demands (self-imposed short total sleep time) or because they are natural short sleepers and require less sleep. ${ }^{9}$

\section{Differential Diagnosis}

Insomnia may be divided into extrinsic and intrinsic disorders. Extrinsic disorders include problems 
with sleep hygiene, substance abuse, and situational stress.

Intrinsic disorders include psychophysiologic insomnia, idiopathic or primary insomnia, obstructive sleep apnea, restless legs syndrome, shift work sleep disorder, and circadian rhythm disorders. Psychophysiologic insomnia occurs after an shortterm stressor. After several nights of poor sleep, the patient focuses on the inability to sleep, which perpetuates the problem. ${ }^{10}$ Circadian rhythm abnormalities include advanced sleep phase disorder and delayed sleep phase disorder. Patients with advanced sleep phase disorder, common in elderly persons, become sleepy in the early evening, sleep a normal amount, and then have very early morning awakening. These patients may struggle to stay awake until a more socially acceptable evening time. Patients with delayed sleep phase disorder, more common in adolescence, may not become sleepy until after midnight, sleep a normal length of time, and awaken very late in the morning. ${ }^{11}$

As one ages, stage 3 and 4 sleep decreases and stage 1 increases, resulting in less restorative sleep. Nocturnal awakenings also increase, causing more fragmented sleep. ${ }^{12}$ Sleep complaints are therefore more common in elderly persons. ${ }^{13}$ Although these changes in sleep patterns may be responsible for perceived insomnia, many sleep complaints in the elderly are caused by medical conditions or medication use that increases with age.

Medical problems such as arthritis, allergies, congestive heart failure, and benign prostatic hypertrophy affect sleep. Physiologic causes for sleep disturbances include hormonal shifts commonly affecting postpartum and perimenopausal women. Medications used to treat many common disorders, such as decongestants, $\beta$-agonists, corticosteroids, $\beta$-blockers, diuretics, antidepressants, and $\mathrm{H}-2$ blockers disturb sleep. ${ }^{11}$ If a medication is thought to be the cause of a sleep disturbance, alternative medications should be considered. If there are no alternatives, medications to treat the insomnia could be added to the patient's regimen.

It can be difficult to distinguish between primary sleep complaints and those associated with psychiatric disorders. Insomnia is often a symptom of underlying anxiety, depression, or panic disorder. A survey of office-based physicians showed that $30 \%$ of patients diagnosed with insomnia were also diagnosed with depression. ${ }^{14}$ Another study found that approximately $40 \%$ of patients presenting to sleep specialists have a psychiatric disorder. ${ }^{15}$ Furthermore, $10 \%$ to $15 \%$ of insomniacs are substance abusers. $^{3}$

The approach to a sleep complaint begins with a thorough sleep history. Sleep-wake patterns should be elicited and confirmed by the patient's bed partner if possible. A family history may be useful. More than $30 \%$ of insomniacs will have a family history of sleep disorders, especially among firstdegree female relatives. ${ }^{16}$ The sleep history should include evaluation of underlying psychiatric disorders, current medication regimens, and associated symptoms that may coexist with the inability to sleep. Previous treatment attempts should also be noted. The physical examination is less useful, although physical evidence of comorbidities, such as allergies or obstructive sleep apnea, may be important.

Polysomnography is not indicated unless a sleep-related breathing disorder is suspected. Patients with daytime sleepiness, snoring, witnessed apneic spells, and a body mass index over 35 have a greater than $70 \%$ probability of having sleep apnea. ${ }^{17}$ Patients with these symptoms, narcolepsy, or sleepwalking should be referred for polysomnography. Additionally, patients who have daytime sleepiness and have such occupations as pilot or truck driver should have sleep studies done.

\section{Nonpharmacologic Treatment}

Treatment of insomnia should be individualized based on the nature and severity of symptoms and should occur after other causes have been considered, diagnosed, and treated. Nonpharmacologic treatment is less expensive and has fewer side effects compared with pharmacologic treatment. Pharmacologic treatment should be used if the patient will benefit from the more rapid effect of drug therapies while pursuing the longer-lasting effects of behavior modifications.

Nonpharmacologic treatments for insomnia are considered effective if they decrease sleep onset latency or increase total sleep time by 30 minutes. Most treatment studies use patient-reported sleep diaries to measure outcome. Criteria used include total sleep time, sleep-onset latency, and number of nocturnal awakenings. A meta-analysis of 48 individual studies of behavioral therapy found stimulus control therapy to be effective and to be superior to progressive relaxation, imagery training, and para- 
doxical intention. Progressive muscle relaxation is superior to placebo. A more recent meta-analysis compared the outcomes of pharmacotherapy and behavioral therapy and found no differences between the 2 except that behavioral therapy more significantly decreased sleep latency. ${ }^{18}$

Stimulus control therapy, based on the premise that insomnia is a conditioned response to temporal and environmental stimuli normally associated with sleep, reassociates the bed with sleep and is especially effective for sleep-onset insomnia. ${ }^{13,19}$ The patient is instructed to leave the bedroom if unable to fall asleep within 20 minutes. The patient then returns to bed only when very sleepy, repeating the cycle as often as needed through the night. Patients avoid sleep incompatible activities when in bed (reading or watching television).

With paradoxical intention therapy, the patient confronts their fear of sleeplessness by staying awake. This decreases concern about the consequences of lack of sleep and decreases performance anxiety about falling asleep. Patients who undergo cognitive therapy do not necessarily improve their sleep parameters, but they have increased satisfaction with their sleep patterns. ${ }^{13}$

Relaxation therapies, based on the premise that the aroused state precludes sleep, are effective, especially in young adults, but less so than stimulus control or sleep restriction. ${ }^{13}$ Relaxation training includes progressive muscle relaxation, where patients decrease muscle tension by tensing and relaxing different muscle groups. When using imagery training to decrease cognitive arousal, patients focus on pleasant or neutral images or use thoughtstopping techniques.

A recent study showed cognitive behavioral therapy decreased sleep latency by $54 \%$, compared with $16 \%$ with relaxation therapy and $12 \%$ with placebo treatment. ${ }^{19}$ Cognitive therapy addresses dysfunctional beliefs; patients may have unrealistic expectations for sleep and misconceptions about causes and consequences of their insomnia. Cognitive therapy is as effective as pharmacologic therapy in the elderly. ${ }^{20}$ Many insomniacs develop anxiety about going to sleep and a fear of sleeplessness, which may perpetuate the insomnia. Others nap to compensate for poor nocturnal sleep, but this aggravates the insomnia.

Sleep restriction and temporal control are probably beneficial. Sleep restriction therapy attempts to reassociate the bed with sleep. This therapy pre- sumes that insomniacs spend more time in bed in an attempt to get more sleep, thereby decreasing sleep efficiency and increasing frustration and perception of insomnia. Sleep restriction increases sleep efficiency and sleep quality. ${ }^{13}$ Patients decrease the amount of time in bed to their average total sleep time (the amount of time in the bed that they actually sleep), creating a mild sleep deprivation and resulting in better sleep. Each week, when sleep efficiency (time asleep/time in bed $\times 100$ ) exceeds $90 \%$, the patient increases time in bed by 20 minutes. When sleep efficiency is less than $80 \%$, time in bed is decreased by 20 minutes. ${ }^{13}$ Time in bed should not decrease to below 5 hours. ${ }^{19} \mathrm{Tem}$ poral control therapy requires the patient to get up at the same time each day, regardless of how much they sleep, and to avoid naps. ${ }^{21}$

Poor sleep bygiene may worsen mild insomnia, but improving sleep hygiene alone is unlikely to impact severe insomnia. Improving sleep hygiene involves giving patients a list of instructions facilitating a regular sleep-wake schedule. ${ }^{8}$ Instructions include: participate in a relaxing activity until tired, go to bed only when tired, use the bedroom only for sleep and sexual activity, avoid naps, avoid caffeine, nicotine, and alcohol 4 to 6 hours before bed, avoid poor sleeping environments, decrease fluid intake before bed, avoid heavy meals, heavy exercise, stimulating late-evening activities, and eliminate bedroom clocks. Exercise, unless performed immediately before bedtime, increases sleep quality. Moderate exercise improves sleep quality, onset latency, and duration in older adults. ${ }^{22}$ One study showed that exercise and benzodiazepines (BDZs) were similar in their sleep-improving abilities. ${ }^{23}$

\section{Pharmacologic Treatment}

Prescribing sleep agents should be based on the severity of daytime symptoms and the likelihood that the short-term insomnia, if untreated, could progress into long-term insomnia. ${ }^{11}$ The ideal sleep agent has a quick onset of action to decrease sleep latency, a duration of action that prevents early morning awakenings, and minimal side effects. ${ }^{11}$ The lowest effective dose of an agent should be used. Medications should be used for a short period (2 to 4 weeks) and intermittently, based on the individual patient's return to an acceptable sleep cycle. ${ }^{21}$

L-Tryptophan, though banned in 1989 because of its association with eosinophilia-myalgia syn- 
Table 1. FDA-Approved Medications for the Treatment of Insomia ${ }^{35}$

\begin{tabular}{ll}
\hline Chloral hydrate & Paraldehyde \\
Diphenhydramine & Pentobarbital \\
Doxylamine & Quazepam \\
Estazolam & Secobarbital \\
Ethchlorvynol & Temazepam \\
Ethinamate & Triazolam \\
Flurazepam & Trimeprazine \\
Glutethimide & Zaleplon \\
Methyprylon & Zolpidem \\
\hline
\end{tabular}

drome, has been promoted as a sleep aid. It remains available in a low-dose $(500 \mathrm{mg})$ capsule $^{24}$; milk contains L-tryptophan in its natural form. The safety and efficacy of the lower dose has not been studied.

People with sleep disturbances may drink alcohol to cause drowsiness. In 1 study of insomniacs, $28 \%$ used alcohol to help them sleep, and 67\% found it effective. ${ }^{25}$ However, alcohol can act as a central nervous system (CNS) stimulant and may increase nocturnal awakenings. Using alcohol as a sleep aid should be discouraged because of its abuse potential.

Melatonin, a neurohormone secreted by the pineal gland, has been publicized as a cure for many sleep problems. Nocturnal secretion of melatonin and its suppression by bright light regulate circadian rhythms and sleep. ${ }^{26}$ Sleep disturbances occur when melatonin secretion is dyssynchronous from the light-dark cycle. ${ }^{27}$ Melatonin secretion decreases with age and may be partly responsible for sleep disorders in the elderly. ${ }^{28}$ Tobacco, alcohol, and medications, such as nonsteroidal anti-inflammatory drugs, calcium channel blockers, BDZs, fluoxetine, and steroids decrease melatonin production. $^{27}$

Melatonin is available in 0.3 - to 5 -mg doses. ${ }^{27}$ Doses of more than $1 \mathrm{mg}$ will produce supraphysiologic levels. The half-life of melatonin is only 30 to 50 minutes, ${ }^{27}$ so there is minimal residual effect the next morning. The main side effect is drowsiness. It is unclear whether long-term use suppresses endogenous production. Melatonin can interact with luteinizing hormone and should not be given to prepubertal or pregnant women.

A recent study did not find any improvement in sleep quality or quantity with melatonin administration. ${ }^{29}$ In fact, if given incorrectly, melatonin could exacerbate sleep disturbances. Melatonin given in the early evening will advance the circa- dian phase, ${ }^{28}$ so this dosing regimen could help those patients who have trouble falling asleep but then sleep late in the morning. However, elderly patients who complain of early morning awakenings may have their sleep problem worsened by a prebedtime dose. Conversely, early morning administration of melatonin delays the circadian phase, which might help patients who become sleepy earlier and awaken early.

Valerian root is an over-the-counter herbal medicine used for insomnia. Valerian root, valeriana officinalis, is a perennial plant with a volatile oil found in the rhizome in the dried form. The oil contains 3 distinct compounds, present in various amounts, but all have sedative effects. Compared with BDZs, side effects are generally less common. Sedation is thought to be caused by inhibition of the breakdown of either $\gamma$-aminobutyric acid (GABA) or direct metabolites that have GABA-like properties, thus producing central sedation. Clinical trials using $400 \mathrm{mg}$ of the aqueous extract decreased sleep latency and increased slow wave sleep. ${ }^{30}$ Valerian root is classified as "generally recognized as safe" (GRAS) in the United States for food use and is officially in the European Pharmacopoeia. A recent FDA Advisory ${ }^{31}$ has recommended against the use of kava because of liver toxicity.

The most commonly used nonprescription agents are the antihistamines diphenhydramine and doxylamine. Side effects include dry mouth, constipation, and urinary retention. They are effective in inducing drowsiness when taken at bedtime; however, their half-life of up to 8 hours may cause residual daytime sedation.

$\mathrm{BDZs}$ are frequently prescribed for sleep disturbances. BDZs shorten sleep latency, decrease nocturnal awakenings, and increase total sleep time. ${ }^{32}$ However, they also shorten REM sleep. BDZs have muscle relaxant, anticonvulsant, and anxiolytic properties because of their nonselective binding to BDZ2 and BDZ3 receptor subtypes. ${ }^{32}$

For the elderly and those with renal or hepatic impairment, a BDZ with a short half-life (triazolam) and without active metabolites would be preferable to avoid oversedation from an accumulation of active metabolites. A BDZ with a longer half-life (flurazepam) is appropriate for patients with daytime anxiety. An intermediate agent, such as temazepam or estazolam, may be a reasonable compromise for patients with early morning awakenings. 

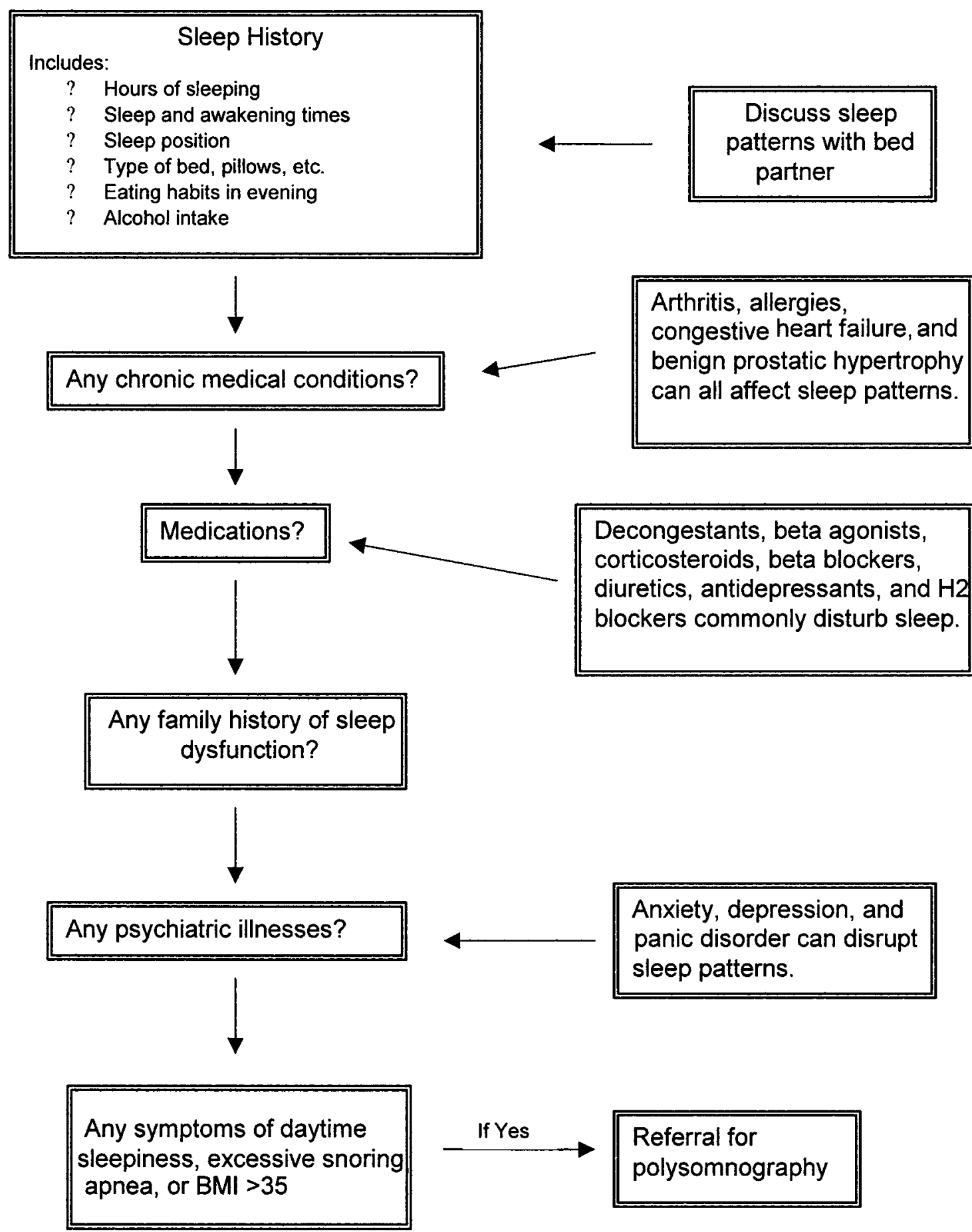

Figure 1. Diagnostic approach to insomnia

BDZ side effects include daytime sedation, anterograde amnesia, respiratory depression, and withdrawal symptoms. Tolerance may develop within 1 to 2 weeks. ${ }^{8}$ BDZs have been associated with rebound insomnia, which can occur after 1 night of BDZ use. ${ }^{32}$ The risk of rebound insomnia is greater with higher doses and short half lives, though unaffected by BDZ use duration. ${ }^{32}$ Gradual discontinuation can minimize rebound insomnia. ${ }^{32}$ Because of their addictive effects, BDZs should be avoided or used with caution for patients with substance abuse tendencies.

Case reports of confusion, amnesia, and behavior problems were reported with the short-acting 


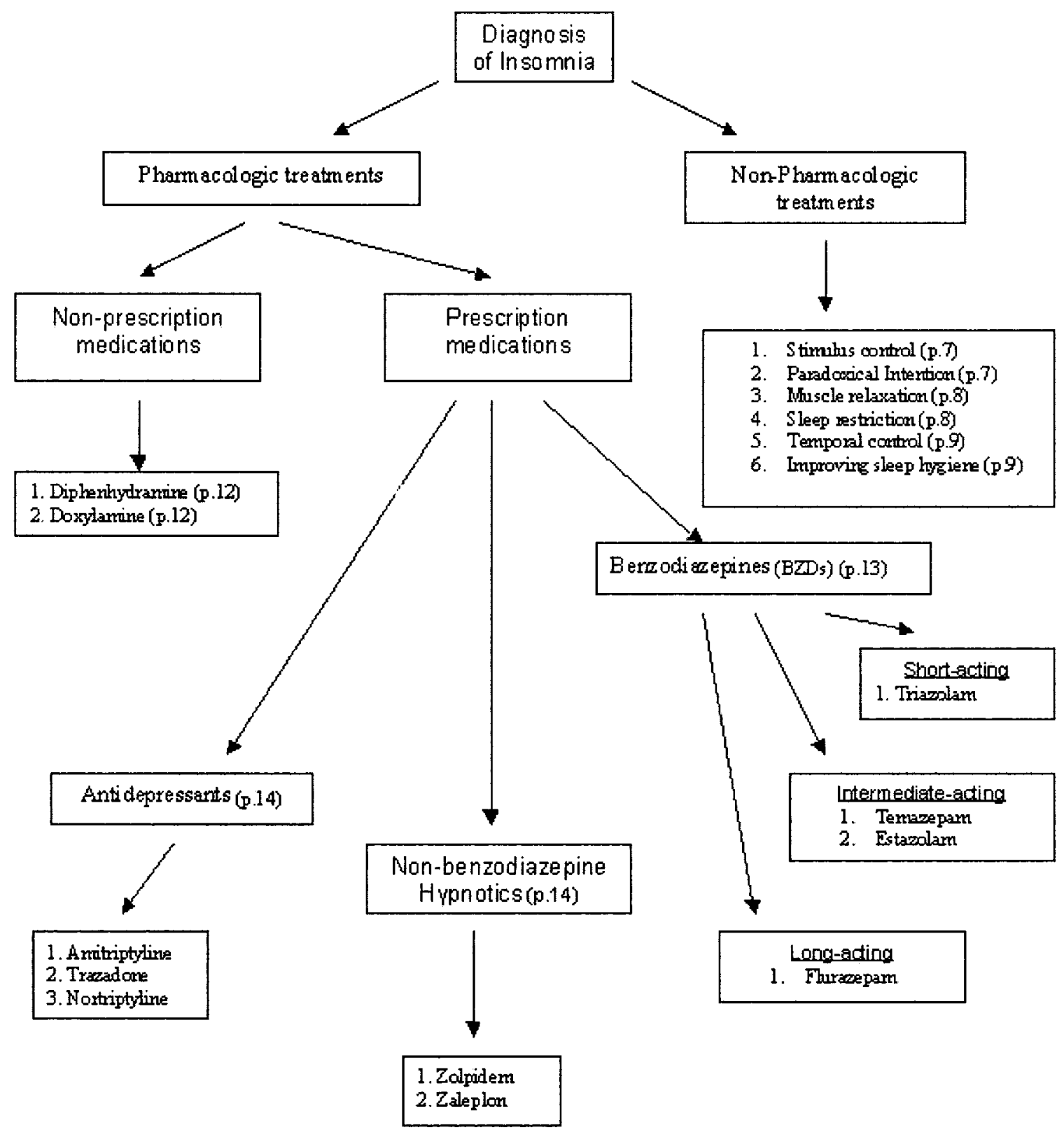

Figure 2. Treatment algorithm

BDZ triazolam, resulting in its removal from the market in Europe in 1991. ${ }^{33}$ Subsequently, prescriptions for BDZs decreased 30\% between 1987 and $1991 .^{32}$

In the place of BDZs, antidepressants with sedation as a side effect, such as amitriptyline, nortriptyline, and trazodone, were increasingly used to treat insomnia. Amitriptyline and nortriptyline were previously used as sleep agents but their usage has decreased because of anticholinergic side effects and the availability of newer agents. Trazodone is widely accepted for the treatment of insomnia in the nondepressed patient even though controlled studies are lacking. Trazodone decreases the insomnia caused by selective serotonin reuptake inhibitors and is a good choice for depressed patients with difficulty sleeping. ${ }^{8}$ Trazodone does not affect sleep latency but does decrease REM sleep and may be associated with significant rebound insomnia. ${ }^{11}$ Priapism is a potentially serious side effect of trazodone and may limit its use in men. Trazodone helps depressed insomniacs more than fluoxetine during the first week of administration, but by the second week of administration, there is no signifi- 
cant difference. ${ }^{34}$ In direct comparison, zolpidem was more effective than trazodone initially, as well as after the first week of treatment. ${ }^{35}$

Marketed since 1993, the most commonly prescribed sleep agents are the selective $\mathrm{BDZ}$ receptor agonists zolpidem and zaleplon. Zolpidem and zaleplon are BDZ1 receptor subtype agonists and are highly selective for the GABA-chloride channel within the type I-BDZ receptors in the CNS. The modulating effects on GABA produce sedation. ${ }^{31}$

Zolpidem has a rapid onset of action and a halflife of only 1.5 to 2.5 hours. ${ }^{8}$ These characteristics allow patients to take zolpidem later in the night when having trouble falling asleep without worrying about residual cognitive impairment the next morning. Zolpidem decreases sleep latency and increases total sleep time; unlike nonselective BDZs, zolpidem does not decrease REM or $\delta$ sleep. ${ }^{8}$

The usual dose of zolpidem is $10 \mathrm{mg}$ at bedtime. This is decreased to $5 \mathrm{mg}$ in patients older than 65 or with hepatic disease. Common side effects include drowsiness $(5 \%)$, dizziness $(5 \%)$, headache (3\%), and gastrointestinal symptoms (4\%). Most side effects are dose-related, occurring at doses above $20 \mathrm{mg}$ per day. One percent to $2 \%$ of patients taking zolpidem complain of memory problems, nightmares, and confusion, and there have been case reports of sensory disturbances and psychotic symptoms. ${ }^{8}$

Zaleplon is another selective BDZ receptor agonist. Patients can take zaleplon as close as 4 hours before waking without residual side effects. ${ }^{31}$ The dose of zaleplon is $10 \mathrm{mg}$ at bedtime, although elderly patients should begin with a $5-\mathrm{mg}$ dose. Zaleplon is a good choice for patients who have initial insomnia. Patients with difficulty staying asleep and who have frequent nocturnal awakenings may benefit from a sleep agent with a longer half-life.

Zoplicone is a selective $\mathrm{BDZ}$ receptor agonist not yet available in the United States.

\section{Summary}

Insomnia is a common medical problem that is associated with decreased work performance, lost productivity, accidents, and hospitalization. Cost estimates exceed $\$ 100$ billion per year. Evaluation and treatment of insomnia should be individualized. Nonpharmacologic treatment is inexpensive and may be effective. Melatonin and plant deriva- tives such as valerian root deserve further research. Tricyclic antidepressants and antihistamines should be used with caution because of their side-effect profiles, especially when used in the elderly or with other medications. Trazodone and nonselective BDZs can be effective insomnia treatments. Newer agents zolpidem and zaleplon are selective benzodiazepine (subtype 1) receptor agonists that have a rapid onset, short half-life, and minimal side effects. Consider using a short-term pharmacologic agent while promoting long-term behavioral changes to improve sleep patterns.

\section{References}

1. The Gallup Organization. Sleep in America: a national survey of US adults. Washington DC: National Sleep Foundation; 1995.

2. Kiley J. Insomnia research and future opportunities. Sleep 1999;22 Suppl 1:S344-5.

3. Meyer TJ. Evaluation and management of insomnia. Hosp Pract (Off Ed) 1998;33:75-8, 83-6.

4. Eddy M, Walbroehl G. Insomnia. Am Fam Physician 1999;59:1911-6.

5. American Psychiatric Association. Diagnostic and statistical manual of mental disorders. 4th ed. Washington DC: American Psychiatric Association; 1994.

6. Wagner J, Wagner M, Hening W. Beyond benzodiazepines: alternative pharmacologic agents for the treatment of insomnia. Ann Pharmacother 1998;32: 680-91.

7. Lacks P, Morin C. Recent advances in the assessment and treatment of insomnia. J Consult Clin Psychol 1992;60:586-94.

8. Diagnostic Classification Steering Committee of the America Sleep Disorders Association. International classification of sleep disorders-diagnostic and coding manual. Rochester $(\mathrm{MN})$ : American Sleep Disorders Association; 1990.

9. Jones C, Czajkowski L. Evaluation and management of insomnia in menopause. Clin Obstet Gynecol 2000;43:184-97.

10. Attarian H. Helping patients who say they cannot sleep. Postgrad Med 2000;7:127-42.

11. Ancolu-Isreal S. Insomnia in the elderly: a review for the primary care practitioner. Sleep 2000;23 Suppl 1:S23-38.

12. Morin C, Mimeault V, Gagne A. Nonpharmacological treatment of late-life insomnia. 1999 J Psychosom Res 1999;46:103-16.

13. Simon G, VonKorff M. Prevalence, burden, and treatment of insomnia in primary care. Am J Psychiatry 1997;154:1167-23.

14. Rakecki S, Brunton A. Management of insomnia in office-based practice. Arch Fam Med 1993;2:112934. 
15. Buysee D, Reynolds C, Kupfer D, et al. Clinical diagnosis in 216 insomnia patients using the International Classification of Sleep Disorders (ICSD), DSM-IV, and ICD-10 categories: a report from the APA/NIMH DSM-IV field trial. Sleep 1994;17:630-7.

16. Bastien C, Morin C. Familial incidence of insomnia. J Sleep Res 2000;9:45-54.

17. American Sleep Disorders Association. Practice parameters for the use of portable recording in the assessment of obstructive sleep apnea. Standards of Practice Committee of the American Sleep Disorders Association. Sleep 1994;17:372-7.

18. Smith M, Perlis M, Park A, et al. Comparative Metaanalysis of pharmacotherapy and behavior therapy for persistent insomia. Am J Psychiatry 2002;159:511.

19. Edinger J, Wohlgemuth W, Radtke R, Marsh G, Quillian R. Cognitive behavioral therapy for treatment of chronic primary insomnia. JAMA 2001;285: 1856-64.

20. Morin C, Colecchi C, Stone J, Sood R, Brink D. Behavioral and pharmacological therapies for latelife insomnia. JAMA 1999;284:991-9.

21. Holbook A, Crowther R, Lotter A, Cheng C, King D. The diagnosis and management of insomnia in clinical practice: a practice evidence-based approach. Can Med Assoc J 2000;162:210-6.

22. King A, Oman R, Brassington G, Bliswise D, Haskell W. Moderate intensity exercise and self-rated quality of sleep in older adults: a randomized controlled trial. JAMA 1997;277:32-7.

23. Morin C, Culbert J, Schwartz S. Nonpharmacological interventions for insomnia: a meta-analysis of treatment efficacy. Am J Psychiatry 1994;151:117280.

24. Hendrix S. Nonestrogen management of menopausal symptoms. Endocrinol Metab Clin North Am 1997;26:379-90.

25. Roehrs T, Papineau K, Rosenthal L, Roth T. Etha- nol as a hypnotic in insomniacs: self administration and effects on sleep and mood. Neuropsychopharmacology 1999;20:279-86.

26. Avery D, Lenz M, Landis C. Guidelines for prescribing melatonin. Ann Med 1998;30:122-30.

27. Shochat T, Haimov I, Lavie P. Melatonin-the key to the gate of sleep. Ann Med 1998;30:81-7.

28. Arendt J, Deacon S. Treatment of circadian rhythm disorders-melatonin. Chronobiol Int 1997;14:185204.

29. Zhdanova IV, Wurtman RJ, Regan MM, Taylor JA, Shi JP, LeClair OU. Melatonin treatment for agerelated insomnia. J Clin Endocrinol Metab 2001;86: 4727-30.

30. Snitker JA, Bell WL, Schweain SL, Short RM, Burnham TH. Valerian. The review of natural products. St. Louis: Facts and Comparisons; 1999. p. 1-2.

31. Consumer advisory: Kava-containing dietary supplements may be associated with severe liver injury. Rockville (MD): Center for Food Safety and Applied Nutrition, US Food and Drug Administration; 2002 [cited 2003 Oct 28]. Available from http://www.cfsan.fda.gov/\% 7Edms/addskava.html.

32. Mitler MM. Nonselective and selective benzodiazepine receptor agonists-where are we today? Sleep 2000;23 Suppl 1:S39-47.

33. Wysowski D, Barash D. Adverse behavioral reactions attributed to triazolam in the Food and Drug Administration's Spontaneous Reporting System. Arch Intern Med 1991;151:3003-8.

34. Kallepalli B, Bhatara V, Fogas B, Tervo R, Misra L. Trazodone is only slightly faster than fluoxetine in relieving insomnia in adolescents with depressive disorders. J Child Adolesc Psychopharmacol 1997;7: 97-107.

35. MICROMEDEX Healthcare Series Integrated Index-Drugdex drug evaluations: FDA labeled useinsomnia. Greenwood Village (CO): Thomson MICROMEDEX; 2003. 